\title{
Study on the mechanism of JAK2/STAT3 signaling pathway-mediated inflammatory reaction after cerebral ischemia
}

\author{
YUQUAN WU ${ }^{1,2^{*}}$, JUAN XU $^{1 *}$, JING XU $^{1}$, WEI ZHENG ${ }^{1}$, QINGYONG CHEN ${ }^{1,2}$ and DEMING JIAO ${ }^{2}$ \\ ${ }^{1}$ Department of Geriatrics, The 117th Hospital of PLA, Hangzhou, Zhejiang $310013 ;{ }^{2}$ Department of Clinical Laboratory, \\ Hangzhou Clinical College of The People's Liberation Army of Anhui Medical University, \\ Hangzhou, Zhejiang 310013, P.R. China
}

Received October 2, 2017; Accepted January 12, 2018

DOI: $10.3892 / \mathrm{mmr} .2018 .8477$

\begin{abstract}
The present study aimed to investigate the mechanism by which the Janus kinase (JAK)2/signal transducer and activator of transcription (STAT)3 signaling pathway mediates cerebral ischemia and the efficacy of pharmaceutical intervention. The rat model of middle cerebral artery occlusion (MCAO) was established and confirmed via assessment of changes in the expression of phosphorylated (p)-JAK2, p-STAT3, high-mobility group box 1 (HMGB1), and inflammatory factors using ELISA and western blot analysis. The effects of JAK2/STAT3 inhibitor and curcumin on the expression of p-JAK2, p-STAT3, HMGB1, and inflammatory factors after cerebral ischemia were observed with ELISA, western blotting and immunohistochemical staining. The concentrations of tumor necrosis factor (TNF)- $\alpha$ and HMGB1 in brain tissue homogenate of MCAO group were significantly higher than in the sham group $(\mathrm{P}<0.01)$. The concentration of $\mathrm{p}-\mathrm{JAK} 2 / \mathrm{JAK} 2$ and p-STAT3/STAT3 in the brain tissue homogenate of MCAO group was significantly higher than in the sham group $(\mathrm{P}<0.05)$. The concentrations of TNF- $\alpha$, interleukin (IL)-1 $\beta$, IL-6, and HMGB1 in the group treated with STAT3 inhibitor (MCAO+ rapamycin), JAK2 inhibitor (MCAO + AG490), and $\mathrm{MCAO}+$ curcumin were significantly lower than in the MCAO group $(\mathrm{P}<0.01)$, as well as the relative content of $\mathrm{p}-\mathrm{JAK} 2 / \mathrm{JAK} 2$ and $\mathrm{p}-\mathrm{STAT} 3 / \mathrm{STAT} 3(\mathrm{P}<0.05)$. Inhibition of the JAK2/STAT3 signaling pathway, such as curcumin can reduce the expression of HMGB1 in brain tissue after cerebral ischemia, which can
\end{abstract}

Correspondence to: Professor Yuquan Wu, Department of Geriatrics, The 117th Hospital of PLA, 14 Ling-yin Road, Hangzhou, Zhejiang 310013, P.R. China

E-mail: hz117wyq@163.com

*Contributed equally

Key words: cerebral ischemia, JAK2/STAT3 signaling pathway, HMGB1, inflammatory response, curcumin significantly reduce the inflammatory response after cerebral ischemia.

\section{Introduction}

Stroke is currently the world's second greatest contributor to death rate and morbidity, among which $75 \%$ patients suffered from ischemic stroke, suggesting that ischemic stroke is type of stroke most likely to severely endanger people's health and safety (1-4). In China, around 2 million new cases of stroke were reported every year, and approximately 1.5 million people died of cerebrovascular disease, which places a heavy burden on both families and society (5). It has become a hot topic in recent years to find effective protective mechanism after cerebral ischemia to reduce neuronal death, to improve neurological function, and to delay the progression of the disease (6-8).

Inflammatory response is considered the main pathophysiological mechanism underlying cerebral ischemia (9). Cerebral ischemia produces endogenous damage associated molecular patterns (DAMPs), activating the corresponding receptors leading to ischemic brain damage (10). High-mobility group box 1 (HMGB1) is an important endogenous DAMPs that significantly elevates early in the early stage of cerebral ischemia, promoting the expression of inflammatory factor (TNF- $\alpha$, IL-1, IL-6), which causes pathological inflammatory response (11-14). Currently, the mechanism by which HMGB1 overexpression is activated after cerebral ischemia has not been sufficiently studied $(15,16)$. The JAK2/STAT3 signaling pathway is involved in a variety of inflammatory and anti-inflammatory signaling pathways and multiple physiological and pathological regulation processes (17). Research showed that the JAK2/STAT3 signaling pathway can be activated after cerebral ischemia, mediating the post-ischemic inflammatory response $(18,19)$. Studies of peripheral macrophages showed that activation of the JAK2/STAT3 signaling pathway activation can induce increased expression of HMGB1, which promotes the release of cytokines such as TNF- $\alpha$, thus inducing the inflammatory reaction (20). However, whether or not the activation of JAK2/STAT3 signaling pathway is associated with an increase in HMGB1 expression during cerebral 
ischemia, so inducing the inflammatory reaction, have not yet been reported.

In the present study, a rat model of middle cerebral artery occlusion (MCAO) was established and confirmed with detection of changes in the expression of p-JAK2, p-STAT3, HMGB1, and inflammatory factor using ELISA and western blot analysis. The effects of JAK2/STAT3 inhibitor and curcumin on the expression of p-JAK2, p-STAT3,HMGB1 and inflammatory factors after cerebral ischemia were observed to assess the mechanism underlying the inflammatory reaction as mediated by the JAK2/STAT3 signaling pathway after cerebral ischemia and the efficacy of clinical intervention.

\section{Materials and methods}

Experimental animals and reagents. Male Sprague-Dawley rats (Shanghai Laboratory Animal Center, China) aged 7-8 weeks and weighing 250-300 $\mathrm{g}$ were used in the experiments. The rats were housed under standard laboratory conditions at a temperature of $20-22^{\circ} \mathrm{C}$ and $12 \mathrm{~h}$ light-dark cycle and given access to food and water. All procedures performed in studies involving animals were in accordance with the ethical standards of the institution or practice at which the studies were conducted. The study protocol was approved by the local Ethics Committee of the 117th Hospital of PLA. Rapamycin, AG490, and curcumin were purchased from Sigma-Aldrich (St. Louis, MO, USA). A TNF- $\alpha$ ELISA Kit, IL-1 $\beta$ ELISA Kit, and IL-6 ELISA Kit were purchased from Beyotime Biotechnology (Haimen, China). Antibodies against HMGB1, JAK2, p-JAK2, STAT3, p-STAT3, and the internal standard GAPDH (all from Affinity Biosciences, Cincinnati, OH, USA) were used for western blot analysis and immunohistochemical (IHC) analysis. The secondary antibody was a ready-to-use goat-anti rabbit (or goat-anti-mouse, or donkey-anti-goat) HRP-IgG dilution purchased from Beyotime Biotechnology (Haimen, China).

Preparation of focal cerebral ischemia model in rats. Rats were fasted overnight before the operation with free access to drinking water. The rats were anesthetized with intraperitoneal injection of anesthetic and fixed in supine position. The hair in the median position of the neck was removed and disinfected, and the skin was covered with dressing. The middle part of the neck was incised to expose the right common carotid artery, internal carotid artery, and extracranial branch (pterygopalatine artery), and the external carotid artery and its branches (occipital artery and its superior thyroid artery). The occipital artery, superior thyroid artery, and pterygopalatine artery were clipped and the occipital artery and superior thyroid artery were sliced off. The distal segment of the external carotid artery was ligated, and with a slipknot at the proximal end. A small hemostatic clamp was used to clamp down the common carotid artery and the internal carotid artery. This was followed by a slight incision with micro scissor on the distal end of external carotid artery (proximal end of ligature). A single-head vein indwelling needle sealed up with heparin was inserted along the small cut all the way to the bifurcation of the common carotid artery. The stylet was removed and the micro hemostatic clamp was removed from the internal carotid artery. After completely drilling the blood and gas, the prepared embolus was slowly injected into the internal carotid artery (preparation of embolus: Collect blood samples and centrifuged at 3,000 rpm for $10 \mathrm{~min}$, followed by addition of $\mathrm{CaCl}_{2}$ and thrombin. The mixture was injected into an anesthesia catheter and the tube was placed in a $37^{\circ} \mathrm{C}$ water bath for $15 \mathrm{~min}$. Then, the gel was sliced into $0.8-1.5 \mathrm{~mm}$-long segments. Then, 4-6 segments were selected and placed in $1 \mathrm{ml}$ PBS solution). After complete injection of the embolus, the micro hemostatic clamp was removed from the internal carotid artery and the blood flow was restored for $1 \mathrm{~min}$. Then, the venous indwelling needle was withdrawn, the external carotid artery was ligated, and the neck skin was stitched. The mice in the sham group were given the same treatment, except no embolus was injected. Twenty-four hours after surgery, the rat was incised in the chest under anesthesia to expose the thoracic cavity. The right atrial appendage was cut open and perfused with physiological saline from the left ventricle until clear fluid flowed out. Then, the ischemic tissue of the right cerebrum was collected to prepare tissue homogenate or paraffin sections with $4^{\circ} \mathrm{C}$ physiological saline in the proportion of $1: 10$ by weight.

ELISA. The rat brain tissue homogenate was prepared and centrifuged at $12,000 \mathrm{x} \mathrm{g}$ and $4^{\circ} \mathrm{C}$ for $15 \mathrm{~min}$. The supernatant was collected. Standard control and tissue homogenate was added separately into the well of coated plate. The plate was covered with plate sealing film and incubated at $37^{\circ} \mathrm{C}$ for $30 \mathrm{~min}$. Then, the plate was washed 5 times, followed by addition of $50 \mu \mathrm{l}$ of ELISA reagents. Repeat the incubation and washing steps, then add coloring agent $50 \mu \mathrm{l}$, coloring agent $\mathrm{B}$ $50 \mu \mathrm{l}$ and finally the termination solution $50 \mu \mathrm{l}$ to stop the reaction. The absorbance was measured in a microplate reader and a histogram was drawn.

Western blotting. Every $20 \mathrm{mg}$ of rat brain tissue homogenate was mixed with $200 \mu$ l lysate. The mixture was treated with homogenizer until complete lysis. Then, the sample was centrifuged at $4^{\circ} \mathrm{C}, 12,000 \mathrm{~g}$ for $15 \mathrm{~min}$. The supernatant was collected to measure the protein concentration with BCA kits (Bio-Rad, Hercules, CA, USA). The denatured protein sample was mixed into the sample buffer and added to the sample lanes. After 70 min electrophoresis, the protein was transferred to PVDF membrane (Millipore, Bedford, MA, USA) and blocked with $1 \mathrm{X}$ TBST solution containing $5 \%$ skim milk for $1 \mathrm{~h}$. This was followed by overnight incubation at $4^{\circ} \mathrm{C}$ with primary antibody, the membranes were washed 4 times with $1 \mathrm{X}$ TBST for $10 \mathrm{~min}$ each time. Then, the membrane was incubated with secondary antibody at room temperature for $1 \mathrm{~h}$, then washed 4 times with $1 \mathrm{X}$ TBST for $10 \mathrm{~min}$ each. Finally the fluorescence was developed, enhanced and fixed with enhanced chemiluminescence ECL kit (Pierce, Rockford, IL, USA).

Immunohistochemistry. Paraffin sections were dewaxed and hydrated and placed in EDTA-containing antigen repair buffer ( $\mathrm{pH}$ 9.0). Antigen retrieval was performed in a microwave oven. The slices were placed in $3 \%$ hydrogen peroxide solution to block endogenous peroxidase and blocked with $5 \%$ BSA solution at room temperature for $30 \mathrm{~min}$. Then, the slices were incubated with primary antibody at $4^{\circ} \mathrm{C}$ overnight, 


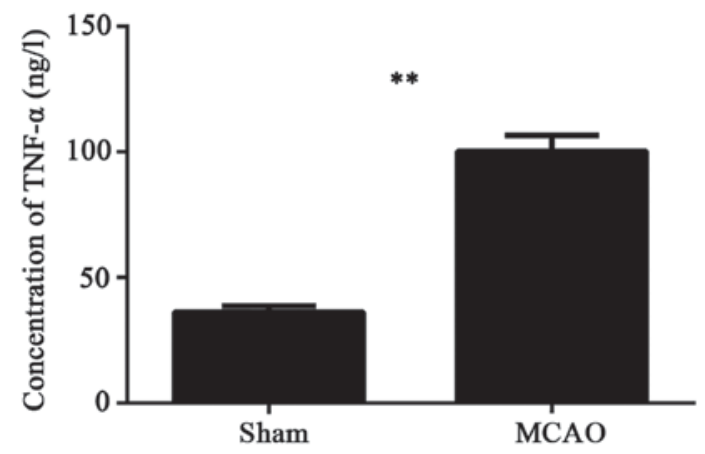

Figure 1. Changes of TNF- $\alpha$ expression in rat brain after cerebral ischemia. Sham group and MCAO group, 5 rats in each group. The brain tissues were harvested $24 \mathrm{~h}$ after the operation, and the TNF- $\alpha$ content in the cerebral tissue homogenate was measured using ELISA $\left({ }^{* *} \mathrm{P}<0.01\right)$.

followed by incubation with secondary antibody for $50 \mathrm{~min}$ at room temperature. Then, the slices were washed with PBS for $15 \mathrm{~min}$. Finally, DAB color developing solution was added dropwise. The development time was controlled by observing the slides under a microscope. Positive staining was indicated with brown spots. The development was terminated by washing with tap water. The nuclei were stained with Harris hematoxylin, and each slice was dehydrated and sealed.

Statistical analysis. Data are expressed as the mean \pm standard error of the mean (SEM) of at least three independent experiments. Standard error bars were included for all data points. Statistical analysis was performed using Student's t-test when only two groups were present or a one-way analysis of variance followed by the Student-Newman-Keuls test when more than two groups were compared. Statistical analyses were conducted with SPSS 17.0 software (SPSS, Inc., Chicago, IL, USA). $\mathrm{P}<0.05$ was considered significant.

\section{Results}

Changes of TNF- $\alpha$ expression in rat brain after cerebral ischemia. Rats were allocated into two groups, a sham group and a MCAO group, with 5 rats in each group. ELISA was performed to detect the TNF- $\alpha$ change in cerebral tissue in each group. As indicated in Fig. 1. The amount of TNF- $\alpha$ in the brain tissue homogenate of MCAO group was significantly higher than in the sham group $(\mathrm{P}<0.01)$.

Changes in HMGB1, JAK2, p-JAK2, STAT3, and p-STAT3 expression in brain tissue of rats after cerebral ischemia. Rats were allocated into two groups, a sham group and MCAO group, with 3 rats in each group. The expression of HMGB1, JAK2, p-JAK2, STAT3, and p-STAT3 were assessed by western blot analysis in the brain tissue of the rats in both groups. As indicated in Fig. 2, the concentration of HMGB1 protein in MCAO group was significantly higher than in the sham group $(\mathrm{P}<0.01)$. The relative levels of $\mathrm{p}-\mathrm{JAK} 2 / \mathrm{JAK} 2$ and p-STAT3/STAT3 in brain tissue homogenate of MCAO group were significantly higher than in the sham group $(\mathrm{P}<0.01)$, suggesting that cerebral ischemia activates the JAK2/STAT3 signaling pathway and promotes HMGB1 overexpression. There remains some question regarding whether the JAK2/STAT3 signaling pathway is involved in HMGB1 induction and its induced inflammatory response.

Effects of rapamycin, AG490, and curcumin on expression of TNF- $\alpha, I L-1 \beta$, and IL- 6 in brain tissue of rats with cerebral ischemia. Rats were allocated into 5 groups of 3 rats each: a cerebral ischemia group (MCAO), inhibitor-treated group (MCAO+saline),STAT3inhibitor-treatedgroup(MCAO+RPM), JAK2 inhibitor-treated group (MCAO + AG490), and curcumin-treated group (MCAO+curcumin). The expression of TNF- $\alpha$, IL-1 $\beta$, and IL- 6 in brain tissue of four groups were detected by ELISA. As indicated in Fig. 3, the concentrations of TNF- $\alpha$, IL-1 $\beta$, and IL- 6 in cerebral tissue homogenate of MCAO group and $\mathrm{MCAO}+$ saline group were comparable $(\mathrm{P}>0.05)$. Compared with MCAO+saline group, the contents of TNF- $\alpha$, IL-1 $\beta$, IL-6 in MCAO+RPM group, MCAO + AG490 group, and $\mathrm{MCAO}+$ curcumin group were significantly lower than in the MCAO+saline group $(\mathrm{P}<0.01)$, suggesting that inhibition of JAK2/STAT3 signaling pathway such as curcumin reduces the release of inflammatory factors after cerebral ischemia.
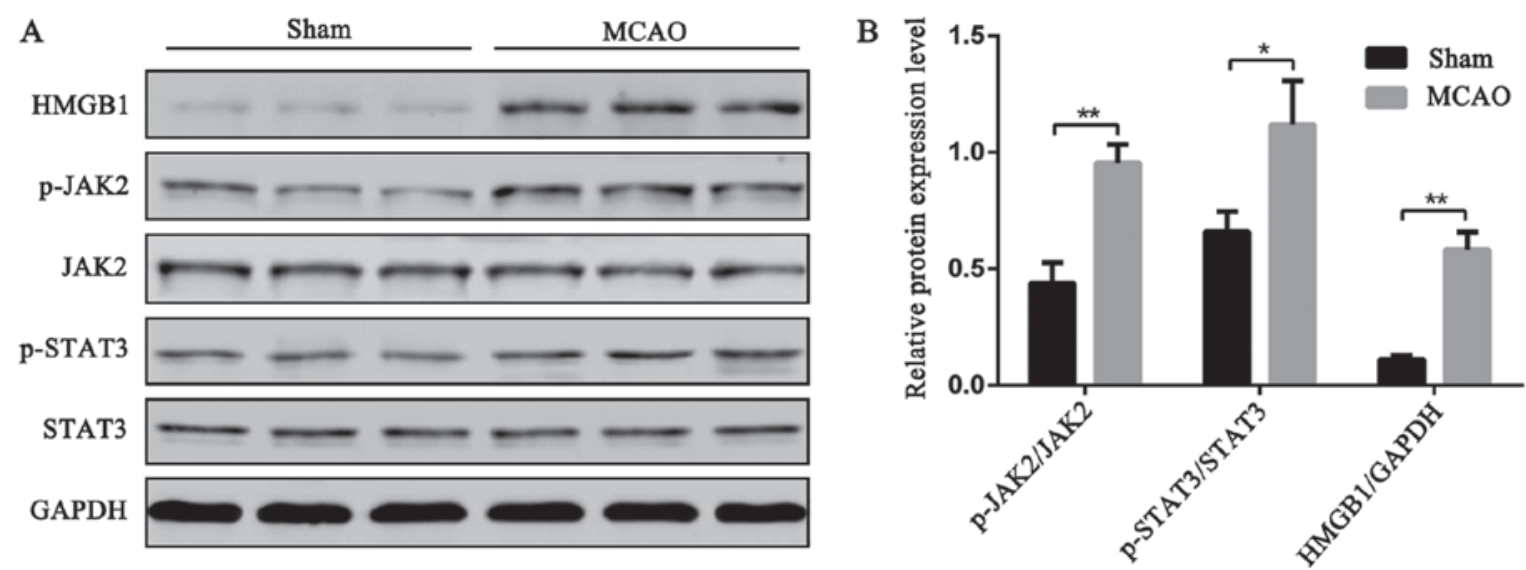

Figure 2. Changes in HMGB1, JAK2, p-JAK2, STAT3, and p-STAT3 expression in brain tissue of rats after cerebral ischemia. Sham group and MCAO group, 3 rats in each group. The brain tissues were harvested $24 \mathrm{~h}$ after operation and change of HMGB1, JAK2, p-JAK2, STAT3, and p-STAT3 contents in the cerebral tissue homogenate was measured using western blotting. (A) Protein bands of cerebral tissue homogenate; (B) Relative expression of the target protein in the homogenate of brain tissue was calculated using the ImageJ image analysis software (Bethesda, MD, USA) $\left(^{* *} \mathrm{P}<0.01,{ }^{*} \mathrm{P}<0.05\right)$. 

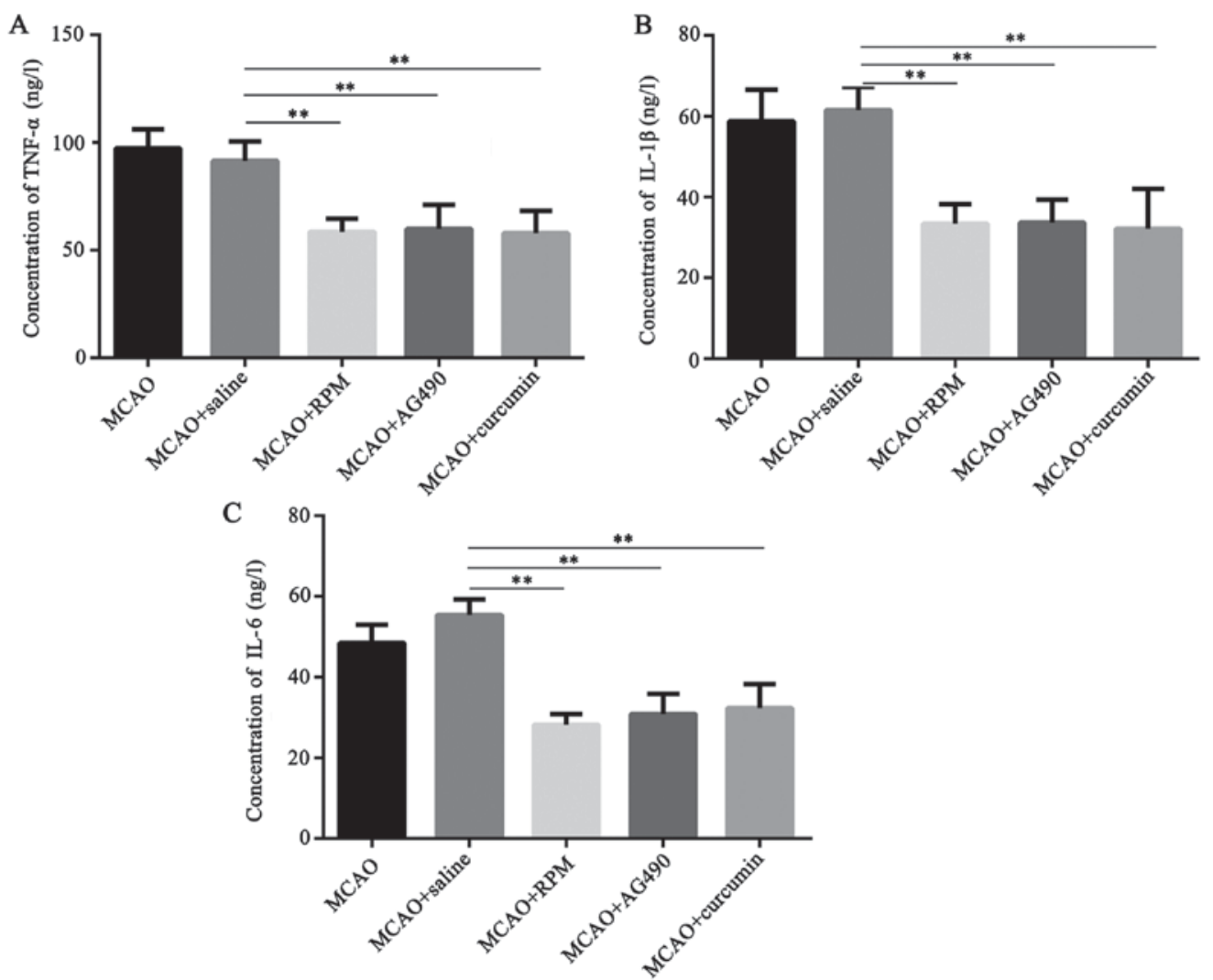

Figure 3. Effects of rapamycin and AG490 on expression of TNF- $\alpha$, IL-1 $\beta$, and IL-6 in brain tissue of rats with cerebral ischemia. MCAO group without pre-treatment before operation; inhibitor-treated group (MCAO+saline group, saline was injected via the tail vein $1 \mathrm{~h}$ before the MCAO operation); STAT3 inhibitor-treated group (MCAO+RPM group, $0.5 \mathrm{mg} / \mathrm{kg}$ rapamycin was injected via the tail vein $1 \mathrm{~h}$ before the MCAO operation); JAK2 inhibitor-treated group (MCAO+AG490 group, $8 \mathrm{mg} / \mathrm{kg}$ AG490 was injected via the tail vein $1 \mathrm{~h}$ before performing MCAO operation); curcumin-treated group (MCAO+curcumin group, $40 \mathrm{mg} / \mathrm{kg}$ curcumin was injected right away via the tail vein after the MCAO operation). 3 rats were treated in each group. The rats were sacrificed $24 \mathrm{~h}$ after the operation and the brain tissue was harvested. The levels of (A) TNF- $\alpha$, (B) IL-1 $\beta$, and (C) IL- 6 in the brain tissue of four groups were detected by ELISA $\left({ }^{* *} \mathrm{P}<0.01\right)$.

A

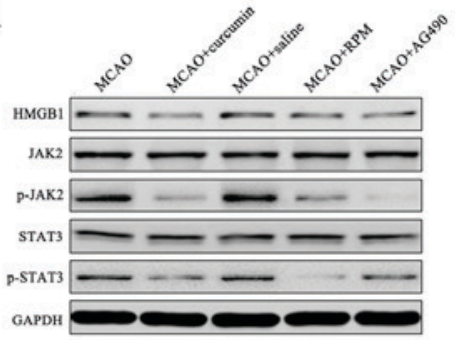

B

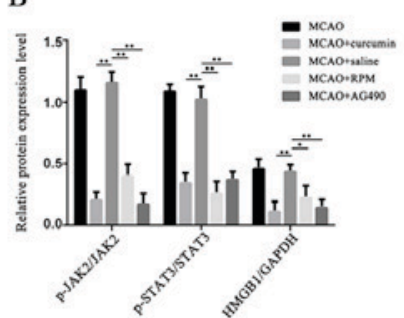

C
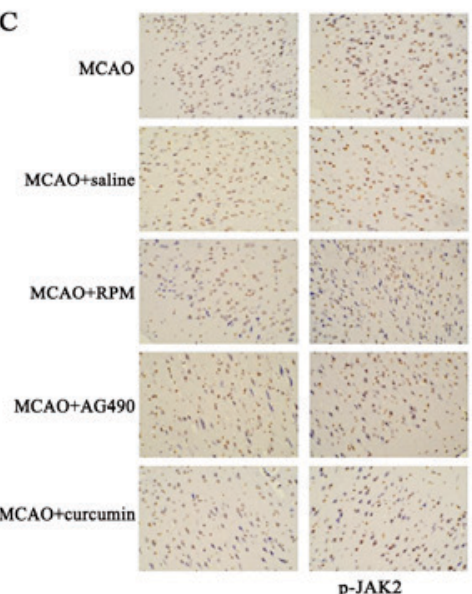
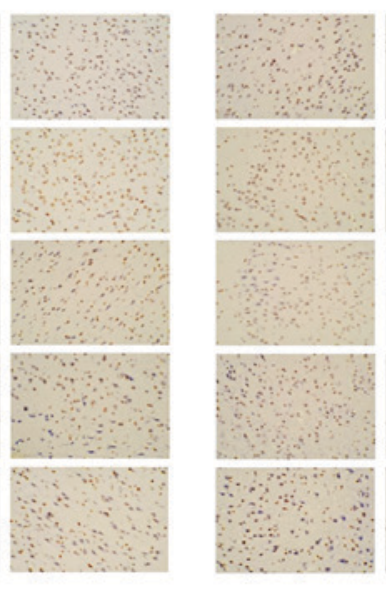
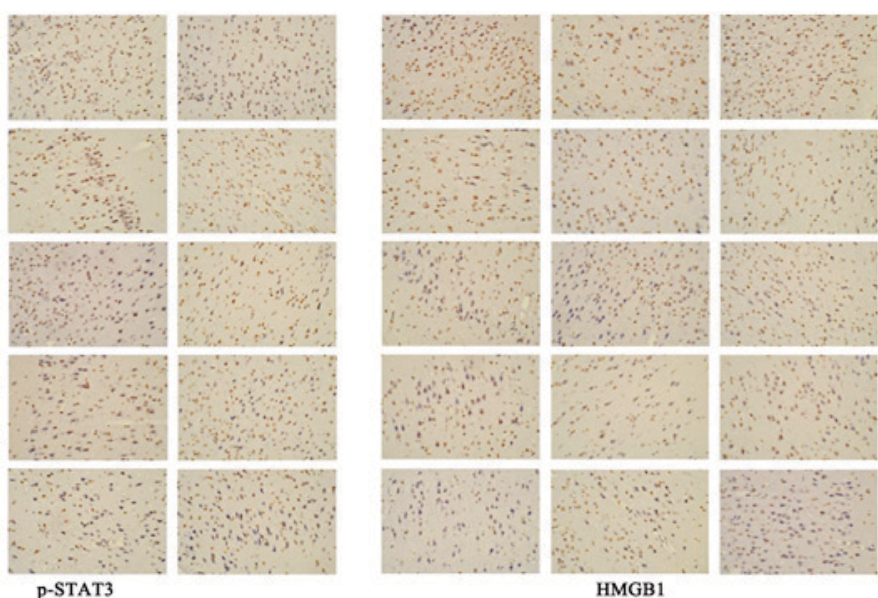

HMGB1

Figure 4. Effects of rapamycin and AG490 on the expression of HMGB1, JAK2, p-JAK2, STAT3, and p-STAT3 in brain tissue of rats with cerebral ischemia Expression of HMGB1, JAK2, p-JAK2, STAT3, and p-STAT3 in the rat brain were detected by (A, B) Western blot analysis and (C) immunohistochemistry (magnification, $\mathrm{x} 200)\left({ }^{*} \mathrm{P}<0.01,{ }^{*} \mathrm{P}<0.05\right)$. 
Effects of rapamycin, AG490, and curcumin on the expression of HMGB1, JAK2, p-JAK2, STAT3, and p-STAT3 in brain tissue of rats with cerebral ischemia. The expression levels of HMGB1, JAK2, p-JAK2, STAT3, and p-STAT3 were detected by Western Blot and immunohistochemistry. As indicated in Fig. 4, the expression of HMGB1, JAK2, p-JAK2, STAT3, and p-STAT3 in the brain tissue homogenate of the $\mathrm{MCAO}+$ saline group was comparable to that of the MCAO group $(\mathrm{P}>0.05)$. The concentrations of $\mathrm{p}-\mathrm{JAK} 2 / \mathrm{JAK} 2$ and p-STAT3/STAT3 in MCAO + RPM group, $\mathrm{MCAO}+\mathrm{AG} 490$ group, and $\mathrm{MCAO}+$ curcumin group were significantly lower than in the MCAO + saline group $(\mathrm{P}<0.01)$, suggesting that inhibition of JAK2/STAT3 signaling pathway such as curcumin can reduce the expression of HMGB1 in ischemic brain tissue.

\section{Discussion}

The pathogenesis of ischemic stroke is very complex. Evidence has shown that inflammation is the most important pathophysiology of cerebral ischemia $(21,22)$. In case of cerebral ischemia, the generation of mediators of inflammation, breakdown of blood-brain barrier, inflammatory cell activation and infiltrate $(23,24)$ can provoke and exacerbate the inflammatory response and lead to brain damage after a series of complex pathological and physiological reactions. HMGB1 plays an important role in the inflammatory cascade after cerebral ischemia and promotes the expression of inflammatory factors (TNF- $\alpha$, IL-1, IL-6), leading to nerve injury and dysfunction (11-14). This study also showed that, in rats with cerebral ischemia, the brain can show high expression of HMGB1 and various inflammatory factors. HMGB1 is essentially a nuclear protein. It is combined with DNA and stored in the nucleus, and it affects the structure of the chromosome to regulate transcription, repair and recombination, and other functions (25). In normal brain tissue, most of the brain cells do not express or express only low levels of HMGB1, only under pathological conditions (ischemia, trauma, etc.) that HMGB1 expression increased by transferring cytoplasm and out to the extracellular area. HMGB1 generates important pro-inflammatory mediators by binding to receptors on the membrane (26). Previous studies have shown that HMGB1 can be activated and significantly increased during the early stage of cerebral ischemia and induce leukocyte infiltration and glial cell activation after ischemia, leading to pathological inflammatory response; while activated inflammatory cells can continue to secrete HMGB1 aggravate inflammatory injury (27). HMGB1 also activates astrocytes in the cerebral ischemic region to secrete MMP-9 to disrupt brain tissue and blood-brain barrier and aggravate the inflammatory response (28). HMGB1 shRNA injection into the striatum can reduce the expression of inflammatory cells in brain tissue, reducing neuronal death. Injection of HMGB1 into the brain tissue of normal mice can induce the expression of inflammatory factors in the tissues (29). The use of anti-HMGB1 neutralizing antibody could significantly reduce the inflammatory reaction and ischemic brain injury (30). In this way, the development of HMGB1 as the target of neuroprotective agents for the clinical treatment of cerebral ischemic injury may provide a new means of intervention.
The mechanism by which HMGB1 becomes activated in patients after ischemic stroke is still not clearly described. JAKs/STATs are important intracellular signal transduction pathways mediated by cytokines, oxidative stress, etc. The JAK protein family includes JAK1, JAK2, JAK3, and TYK2. The STAT protein family includes STAT1, STAT2, STAT3, STAT4, STAT5a, STAT5b, and STAT6 (31-34), among which JAK2/STAT3 is closely related to cerebral ischemia and it can be activated during an early stage of cerebral infraction, thus inducing enhanced expression of pro-inflammatory factors (18,19,35-37). Liu et al reported in a study of peripheral macrophages that activation of the JAK2/STAT3 pathway induced higher expression of HMGB1, HMGB1 further promoting the release of cytokines such as TNF- $\alpha$ (20). Zhang et al found that rapamycin inhibited JAK2/STAT3 signaling pathway to reduce the expression of HMGB1 after acute liver injury, thereby reducing inflammation caused by liver damage (38). Li et al in a study of the intestine, found that activation of JAK2/STAT3 signaling pathway could induce inflammatory reaction, which could be reduced by rapamycin and AG490. However, HMGB1 was not evaluated in the present study (39). In the present research, we found that cerebral expression of HMGB1 and TNF- $\alpha$ would be significantly increased after cerebral ischemia, thus inhibiting the JAK2/STAT3 pathway. Meanwhile, the expression of HMGB1 and inflammatory factors in brain tissue was significantly decreased. We here concluded that inhibition of the JAK2/STAT3 signaling pathway can reduce the expression of HMGB1, thereby significantly reducing the inflammatory response after cerebral ischemia.

Curcumin is a naturally occurring yellow acidic phenol widely found in the rhizoma of turmeric plants such as curcuma longa and curcuma zedoary (40), and has become a research focus given its effects including anti-inflammatory, anti-oxidation, anti-tumor, anti-virus, anti-atherosclerosis and lipid-lowering effects and retarding brain degeneration (41). JAK2/STAT3 signaling pathway is activated after cerebral ischemia, leading to increased expression of HMGB1 and aggravating the postischemic inflammatory responses. In tumor cells and microglia, curcumin has been found to inhibit the activation of JAK2/STAT3 signaling pathway $(42,43)$. Therefore this study also involved curcumin intervention in rats with cerebral ischemia, noting that curcumin could inhibit JAK2/STAT3 signaling pathway and reduce expression of HMGB1 and inflammatory factors. The above findings suggest that curcumin has a protective effect on cerebral ischemic injury, potentially by the mechanism of inhibiting JAK2/STAT3 signaling pathway to reduce HMGB1 expression and alleviate the inflammatory responses.

In conclusion, the JAK2/STAT3 signaling inhibitors such as curcumin have protective effects on cerebral ischemic injury, potentially by the mechanism of reducing HMGB1 expression, thus reducing expression of the inflammatory factors and alleviating the inflammatory responses.

\section{Acknowledgements}

This study was supported by Science and Technology Project of Zhejiang Province (no. 2015C33179) and Medical Scientific Research Fund Project of Nanjing Military Region (no. 14MS147). 


\section{References}

1. Balami JS, Chen RL and Buchan AM: Stroke syndromes and clinical management. QJM 106: 607-615, 2013.

2. Norrving B and Kissela B: The global burden of stroke and need for a continuum of care. Neurology 80 (3 Suppl 2): S5-S12, 2013

3. Meschia JF and Brott T: Ischemic stroke. Eur J Neurol, 2017 (Epub ahead of print)

4. Zevallos J, Santiago F, Gonzalez J, Rodriguez A, Pericchi L, Rodriguez-Mercado R and Nobo U: Burden of stroke in Puerto Rico. Int J Stroke 10: 117-119, 2015.

5. Liu M, Wu B, Wang WZ, Lee LM, Zhang SH and Kong LZ: Stroke in China: Epidemiology, prevention and management strategies. Lancet Neural 6: 456-458, 2007.

6. Lo EH, Dalkara T and Moskowitz MA: Mechanisms, challenges and opportunities in stroke. Nat Rev Neurosci 4: 399-415, 2003.

7. Liu J, Wang Y, Akamatsu Y, Lee CC, Stetler RA, Lawton MT and Yang GY: Vascular remodeling after ischemic stroke: mechanisms and therapeutic potentials. Prog Neurobiol 115 138-156, 2014.

8. Hachinski V, Donnan GA, Gorelick PB, Hacke W, Cramer SC Kaste M, Fisher M, Brainin M, Buchan AM, Lo EH, et al: Stroke: Working toward a prioritized world agenda. Stroke 41 1084-1099, 2010

9. Dirnagl U, Iadecola C and Moskowitz MA: Pathobiology of ischaemic stroke: An integrated view. Trends Neurosci 22: 391-397, 1999.

10. Khoshnam SE, Winlow W, Farzaneh M, Farbood Y and Moghaddam HF: Pathogenic mechanisms following ischemic stroke. Neurol Sci 38: 1167-1186, 2017.

11. Xu M, Zhou GM, Wang LH, Zhu L, Liu JM, Wang XD, Li HT and Chen L: Inhibiting high-mobility group box 1 (HMGB1) attenuates inflammatory cytokine expression and neurological deficit in ischemic brain injury following cardiac arrest in rats. Inflammation 39: 1594-1602, 2016.

12. Wang C, Jiang J, Zhang X, Song L, Sun K and Xu R: Inhibiting HMGB1 reduces cerebral ischemia reperfusion injury in diabetic mice. Inflammation 39: 1862-1870, 2016.

13. Ooboshi H and Shichita T: DAMPs (damage-associated molecular patterns) and inflammation. Nihon Rinsho 74: 573-578, 2016 (In Japanese).

14. Singh V, Roth S, Veltkamp R and Liesz A: HMGB1 as a key mediator of immune mechanisms in ischemic stroke. Antioxid Redox Signal 24: 635-651, 2016.

15. Murphy J: Pharmacological treatment of acute ischemic stroke. Crit Care Nurs Q 26: 276-282, 2003

16. Sun K, Fan J and Han J: Ameliorating effects of traditional Chinese medicine preparation, Chinese materia medica and active compounds on ischemia/reperfusion-induced cerebral microcirculatory disturbances and neuron damage. Acta Pharm Sin B 5: 8-24, 2015.

17. Rawlings JS, Rosler KM and Harrison DA: The JAK/STAT signaling pathway. J Cell Sci 117: 1281-1283, 2004.

18. Satriotomo I, Bowen KK and Vemuganti R: JAK2 and STAT3 activation contributes to neuronal damage following transient focal cerebral ischemia. J Neurochem 98: 1353-1368, 2006.

19. Xie HF, Xu RX, Wei JP, Jiang XD and Liu ZH: P-JAK2 and P-STAT3 protein expression and cell apoptosis following focal cerebral ischemia-reperfusion injury in rats. Nan Fang Yi Ke Da Xue Xue Bao 27: 208-211, 2007 (In Chinese).

20. Liu H, Yao YM, Yu Y, Dong N, Yin HN and Sheng ZY: Role of Janus kinase/signal transducer and activator of transcription pathway in regulation of expression and inflammation-promoting activity of high mobility group box protein 1 in rat peritoneal macrophages. Shock 27: 55-60, 2007.

21. Broughton BR, Reutens DC and Sobey CG: Apoptotic mechanisms after cerebral ischemia. Stroke 40: e331-e339, 2009.

22. Vidale S, Consoli A, Arnaboldi M and Consoli D: Postischemic inflammation in acute stroke. J Clin Neurol 13: 1-9, 2017.

23. Tuttolomondo A, Pecoraro R, Casuccio A, Di Raimondo D, Buttà C, Clemente G, Della Corte V, Guggino G, Arnao V, Maida C, et al: Peripheral frequency of $\mathrm{CD}^{+} \mathrm{CD} 28$-cells in acute ischemic stroke: Relationship with stroke subtype and severity markers. Medicine 94: e813, 2015.

24. Tuttolomondo A, Pedone C, Pinto A, Di Raimondo D, Fernandez P, Di Sciacca R and Licata G; Gruppo Italiano di Farmacoepidemiologia dell'Anziano (GIFA) researchers: Predictors of outcome in acute ischemic cerebrovascular syndromes: The GIFA study. Int J Cardiol 125: 391-396, 2008.
25. Ueda T and Yoshida M: HMGB proteins and transcriptional regulation. Biochim Biophys Acta 1799: 114-118, 2010.

26. Harris HE, Andersson U and Pisetsky DS: HMGB1: A multifunctional alarm in driving autoimmune and inflammatory disease. Nat Rev Rheumatol 8: 195-202, 2012.

27. Muhammad S, Barakat W, Stoyanov S, Murikinati S, Yang H, Tracey KJ, Bendszus M, Rossetti G, Nawroth PP, Bierhaus A and Schwaninger M: The HMGB1 receptor RAGE mediates ischemic brain damage. J Neurosci 28: 12023-12031, 2008.

28. Svedin P, Hagberg H, Sävman K, Zhu C and Mallard C: Matrix metalloproteimse-gene knock-out protects theimrnatare train alter cerebral hypoxia-ischema. J Neurosci 27: 1511-1518, 2007.

29. Kim JB, Sig Choi J, Yu YM, Nam K, Piao CS, Kim SW, Lee MH, Han PL, Park JS and Lee JK: HMGB1, a novel cytokine-like mediator linking acute neuronal death and delayed neuroinflammation in the postischemic brain. J Neurosci 26: 6413-6421, 2006.

30. Liu K, Mori S, Takahashi HK, Tomono Y, Wake H, Kanke T, Sato Y, Hiraga N, Adachi N, Yoshino T and Nishibori M: Anti-high mobility group box 1 monoclonal antibody ameliorates brain infarction induced by transient ischemia in rats. FASEB J 21: 3904-3916, 2007.

31. Babon JJ, Lucet IS, Murphy JM, Nicola NA and Varghese LN: The molecular regulation of Janus kinase (JAK) activation. Biochem J 462: 1-13, 2014.

32. Chai HT, Yip HK, Sun CK, Hsu SY and Leu S: AG490 suppresses EPO-mediated activation of JAK2-STAT but enhances blood flow recovery in rats with critical limb ischemia. J Inflamm 13: 18, 2016.

33. Tao Z, Cheng M, Wang SC, Lv W, Hu HQ, Li CF and Cao BZ: JAK2/STAT3 pathway mediating inflammatory responses in heatstroke-induced rats. Int J Clin Exp Pathol 8: 6732-6739, 2015.

34. Ghoreschi K, Laurence A and O'Shea JJ: Janus kinases in immune cell signaling. Immunol Rev 228: 273-287, 2009.

35. Du W, Hong J, Wang YC, Zhang YJ, Wang P, Su WY, Lin YW, Lu R, Zou WP, Xiong H and Fang JY: Inhibition of JAK2/STAT3 signalling induces colorectal cancer cell apoptosis via mitochondrial pathway. J Cell Mol Med 16: 1878-1888, 2012.

36. Zhang HH, Kuang S, Wang Y, Sun XX, Gu Y, Hu LH and Yu Q: Bigelovin inhibits STAT3 signaling by inactivating JAK2 and induces apoptosis in human cancer cells. Acta Pharmacol Sin 36: 507-516, 2015.

37. Planas AM, Gorina R and Chamorro A: Signalling pathways mediating inflammatory responses in brain ischemia. Biochem Soc Trans 34: 1267-1270, 2006.

38. Zhang Y, Zhao ZF, Han DW, Wang F, Xu RL and Liu MS Rapamycin-induced inhibition of Janus Kinase/signal transducer and activator of transcription pathway affects expression of high-mobility group box 1 in rats with acute liver injury. World Chin J Dig 14: 1916-1920, 2006.

39. Li Y, Li KH, Wen SH, Li C, Li YS, Liu Y, Zhang XY, Yao X and Liu KX: Role of JAK/STAT in intestinal injury induced by intestinal ischemia/reperfusion in rats. Chin J Pathophysiol 27: 2338-2344, 2011

40. Sharma RA, Gescher AJ and Steward WP: Curcumin: The story so far. Eur J Cancer 41: 1955-1968, 2005.

41. Esatbeyoglu T, Huebbe P, Ernst IM, Chin D, Wagner AE and Rimbach G: Curcumin-from molecule to biological function. Angew Chem Int Ed Engl 51: 5308-5332, 2012.

42. Weissenberger J, Priester M, Bernreuther C, Rakel S, Glatzel M, Seifert V and Kögel D: Dietary curcumin attenuates glioma growth in a syngeneic mouse model by inhibition of the JAK1,2/STAT3 signaling pathway. Clin Cancer Res 16: 5781-5795, 2010.

43. Saydmohammed M, Joseph D and Syed V: Curcumin suppresses constitutive activation of STAT-3 by up-regulating protein inhibitor of activated STAT-3 (PIAS-3) in ovarian and endometrial cancer cells. J Cell Biochem 110: 447-456, 2010.

This work is licensed under a Creative Commons Attribution-NonCommercial-NoDerivatives 4.0 International (CC BY-NC-ND 4.0) License. 